\title{
A non-existence result on Cameron-Liebler line classes
}

\author{
J. De Beule, A. Hallez* and L. Storme
}

April 30, 2007

\begin{abstract}
Cameron-Liebler line classes are sets of lines in $\mathrm{PG}(3, q)$ that contain a fixed number $x$ of lines of every spread. Cameron and Liebler classified Cameron-Liebler line classes for $x \in\left\{0,1,2, q^{2}-1, q^{2}, q^{2}+1\right\}$ and conjectured that no others exist. This conjecture was disproven by Drudge for $q=3$ [8] and his counterexample was generalised to a counterexample for any odd $q$ by Bruen and Drudge [4]. A counterexample for $q$ even was found by Govaerts and Penttila [9]. Non-existence results on Cameron-Liebler line classes were found for different values of $x$. In this paper, we improve the non-existence results on Cameron-Liebler line classes of Govaerts and Storme [11], for $q$ not a prime. We prove the non-existence of Cameron-Liebler line classes for $3 \leq x<\frac{q}{2}$.
\end{abstract}

\section{Introduction}

Cameron-Liebler line classes were introduced by Cameron and Liebler [5] in an attempt to classify collineation groups of $\mathrm{PG}(n, q)$ that have equally many point orbits and line orbits. In their paper, they conjectured which groups these are. It is now known [2] that the conjecture is true when the group is irreducible, but there is no classification yet of Cameron-Liebler line classes. In this paper, new non-existence results are presented.

There are many equivalent definitions for Cameron-Liebler line classes. Following Penttila [15], a clique in $\operatorname{PG}(3, q)$ is either the set of all lines through a point $P$, denoted by $\operatorname{star}(P)$, or dually the set of all lines in a plane $\pi$, denoted by line $(\pi)$. The planar pencil of lines in a plane $\pi$ through a point $P$ is denoted by pen $(P, \pi)$.

Definition 1.1 (Cameron and Liebler [5], Penttila [15]) Let $\mathcal{L}$ be a set of lines in $\operatorname{PG}(3, q)$ and let $\chi_{\mathcal{L}}$ be its characteristic function. Then $\mathcal{L}$ is called a Cameron-Liebler line class if one of the following equivalent conditions is satisfied.

*The research of the second author is supported by a research grant of the Research council of Ghent University. 
1. There exists an integer $x$ such that $|\mathcal{L} \cap \mathcal{S}|=x$ for all spreads $\mathcal{S}$.

2. There exists an integer $x$ such that for every incident point-plane pair $(P, \pi)$

$$
|\operatorname{star}(P) \cap \mathcal{L}|+\mid \text { line }(\pi) \cap \mathcal{L}|=x+(q+1)| \operatorname{pen}(P, \pi) \cap \mathcal{L} \mid .
$$

3. There exists an integer $x$ such that for every line $l$ of $\mathrm{PG}(3, q)$

$$
\mid\{m \in \mathcal{L}: m \text { meets } l, m \neq l\} \mid=(q+1) x+\left(q^{2}-1\right) \chi_{\mathcal{L}}(l) .
$$

The parameter $x$ is called the parameter of the Cameron-Liebler line class. We note that the first definition implies that $x \in\left\{0,1,2, \ldots, q^{2}+1\right\}$. Cameron and Liebler [5] showed that a Cameron-Liebler line class of parameter $x$ consists of $x\left(q^{2}+q+1\right)$ lines and that the only Cameron-Liebler line classes for $x=1$ are the cliques, i.e., all lines through a point or all lines in a plane, and for $x=2$ the unions of two disjoint cliques. They also noted that the complement of a Cameron-Liebler line class with parameter $x$ is a Cameron-Liebler line class with parameter $q^{2}+1-x$. So, it suffices to study Cameron-Liebler line classes with parameter $x \leq\left\lfloor\left(q^{2}+1\right) / 2\right\rfloor$. Thus, the case $q=2$ was immediately solved. In their paper, Cameron and Liebler conjectured that no other Cameron-Liebler line classes exist.

Penttila [15] shows that for $q \neq 2$ there exist no Cameron-Liebler line classes with parameter $x=3$ or $x=4$, with possible exception of the cases $(x, q) \in\{(4,3),(4,4)\}$. Bruen and Drudge [3] prove the non-existence of Cameron-Liebler line classes with parameter $2<x \leq \sqrt{q}$. Drudge [8] excludes the existence of a Cameron-Liebler line class with parameter $x=4$ in $\mathrm{PG}(3,3)$, and proves that for $q \neq 2$ there exist no Cameron-Liebler line classes with parameter $2<x \leq \epsilon$, where $q+1+\epsilon$ denotes the size of the smallest nontrivial blocking sets in $\mathrm{PG}(2, q)$. He also gives a counterexample to the conjecture of Cameron and Liebler: a Cameron-Liebler line class with parameter $x=5$ in $\mathrm{PG}(3,3)$, in this way settling the case $q=3$. Bruen and Drudge [4] then construct a Cameron-Liebler line class with parameter $x=\left(q^{2}+1\right) / 2$ for any odd $q$. In [9], Govaerts and Penttila completed the study of the case $x=4$ by showing that there exists no Cameron-Liebler line class with parameter $x=4$ in $\mathrm{PG}(3,4)$. In [9], Govaerts and Penttila also disproved the conjecture of Cameron and Liebler for $q$ even by showing the existence of a Cameron-Liebler line class with parameter $x=7$ in $\mathrm{PG}(3,4)$.

In this paper, new bounds on $x$ for the non-existence of Cameron-Liebler line classes with parameter $x$ are obtained. We improve the results of Govaerts and Storme for $q$ not prime. They proved the following two theorems and corollary [11].

Theorem 1.2 In $\mathrm{PG}(3, q)$, $q$ prime, $q>2$, there exist no Cameron-Liebler line classes with parameter $2<x \leq q$.

Theorem 1.3 (1) In $\mathrm{PG}(3, q)$, q square, there exist no Cameron-Liebler line classes with parameter $2<x \leq \min \left(\epsilon^{\prime}, q^{3 / 4}\right)$, where $q+1+\epsilon^{\prime}$ denotes the size of the smallest nontrivial blocking sets in $\mathrm{PG}(2, q)$ not containing a Baer subplane.

(2) Let $q=p^{3 h}, p \geq 7$ prime, $h \geq 1$ odd, and let $q+1+\epsilon^{\prime \prime}$ denote the size of the smallest nontrivial blocking sets in $\mathrm{PG}(2, q)$ containing neither a minimal blocking set of 
size $q+p^{2 h}+1$, nor one of size $q+p^{2 h}+p^{h}+1$. In $\mathrm{PG}(3, q)$, there exist no Cameron-Liebler line classes with parameter $2<x \leq \min \left(\epsilon^{\prime \prime}, q^{5 / 6}\right)$.

(3) Let $q=p^{3 h}, p \geq 7$ prime, $h>1$ even, and let $q+1+\epsilon^{\prime \prime}$ denote the size of the smallest nontrivial blocking sets in $\mathrm{PG}(2, q)$ containing neither a Baer subplane, nor a minimal blocking set of size $q+p^{2 h}+1$, nor one of size $q+p^{2 h}+p^{h}+1$. In $\mathrm{PG}(3, q)$, there exist no Cameron-Liebler line classes with parameter $2<x \leq \min \left(\epsilon^{\prime \prime}, q^{3 / 4}\right)$.

Corollary 1.4 (1) Let $q$ be a square, $q=p^{h}$, p prime.

1. If $q>16$, then there exist no Cameron-Liebler line classes in $\mathrm{PG}(3, q)$ with parameter $2<x \leq c_{p} q^{2 / 3}$, where $c_{p}$ equals $2^{-1 / 3}$ when $p \in\{2,3\}$ and 1 when $p \geq 5$.

2. If $p>3$ and $h=2$, then there exist no Cameron-Liebler line classes in $\mathrm{PG}(3, q)$ with parameter $2<x \leq q^{3 / 4}$.

(2) Let $q=p^{3}, p \geq 7$ prime, then there exist no Cameron-Liebler line classes in $\mathrm{PG}(3, q)$ with parameter $2<x \leq q^{5 / 6}$.

(3) Let $q=p^{6}, p \geq 7$ prime, then there exist no Cameron-Liebler line classes in $\mathrm{PG}(3, q)$ with parameter $2<x \leq q^{3 / 4}$.

We improve these results for $q$ not prime. Theorem 4.2 gives a new improved bound for general $q \neq 2, q$ not prime.

This theorem will be proven by studying how the lines of the Cameron-Liebler line class with parameter $x$ correspond with $x$-tight sets on $\mathrm{Q}^{+}(5, q)$ and $\left\{x\left(q^{2}+q+1\right), x(q+1) ; 5, q\right\}$ minihypers contained in the Klein quadric $\mathrm{Q}^{+}(5, q)$. Using properties of the associated $\left\{x\left(q^{2}+q+1\right), x(q+1) ; 5, q\right\}$-minihyper combined with the fact that this minihyper lives on $\mathrm{Q}^{+}(5, q)$, gives us new non-existence results on Cameron-Liebler line classes.

\section{Definitions and preliminary results}

Let $v_{n+1}=\left(q^{n+1}-1\right) /(q-1)$ denote the number of points of $\operatorname{PG}(n, q)$.

An $i$-tight set of a finite generalised quadrangle was introduced by Payne $[13,14]$ and was generalised to polar spaces of higher rank by Drudge [7].

Definition 2.1 A set of points $\mathcal{T}$ of a finite polar space of rank $r \geqslant 2$ over a finite field of order $q$ is $i$-tight if

$$
\left|P^{\perp} \cap \mathcal{T}\right|= \begin{cases}i \frac{q^{r-1}-1}{q-1}+q^{r-1} & \text { if } P \in \mathcal{T} \\ i \frac{q^{r^{-1}}-1}{q-1} & \text { if } P \notin \mathcal{T}\end{cases}
$$

This definition poses restrictions on the intersection of a hyperplane with a point set. This has a lot in common with the concept of the minihypers.

Definition 2.2 An $\{f, m ; n, q\}$-minihyper is a pair $(F, w)$, where $F$ is a subset of the point set of $\operatorname{PG}(n, q)$ and $w$ is a weight function $w: \operatorname{PG}(n, q) \rightarrow \mathbb{N}: P \mapsto w(P)$, satisfying

1. $w(P)>0 \Leftrightarrow P \in F$,

2. $\sum_{P \in F} w(P)=f$, and 
3. $\min \left\{\sum_{P \in H} w(P): H\right.$ is a hyperplane $\}=m$.

The weight function $w$ determines the set $F$ completely. When this function has only the values 0 and 1 , then $(F, w)$ is determined completely by the set $F$. In this paper, this will always be the case, so we will not make any further reference to the weight function $w$.

In this paper, we are interested in the $\left\{x\left(q^{2}+q+1\right), x(q+1) ; 5, q\right\}$-minihypers contained in the Klein quadric $\mathrm{Q}^{+}(5, q)$, and associated with the Cameron-Liebler line classes with parameter $x$. The following results discuss the intersections of subspaces with these minihypers. They will be very crucial to prove the improved results on the non-existence of Cameron-Liebler line classes. The first theorem is stated as a corollary in [6].

Theorem 2.3 Let $F$ be a $\left\{\sum_{i=0}^{n-1} \epsilon_{i} v_{i+1}, \sum_{i=1}^{n-1} \epsilon_{i} v_{i} ; n, q\right\}$-minihyper, where $q>h, 0 \leqslant \epsilon_{i} \leqslant$ $q-1,0 \leq i \leq n-1, \sum_{i=0}^{n-1} \epsilon_{i}=h$.

Then a plane of $P G(n, q)$ is either contained in $F$ or intersects it in an $\left\{m_{1}(q+1)+\right.$ $\left.m_{0}, m_{1} ; 2, q\right\}$-minihyper, where $m_{1}+m_{0} \leqslant h$.

Theorem 2.4 (Hamada [12]) Let $F$ be a $\left\{\sum_{i=0}^{n-1} \epsilon_{i} v_{i+1}, \sum_{i=1}^{n-1} \epsilon_{i} v_{i} ; n, q\right\}$-minihyper, where $0 \leqslant \epsilon_{i} \leqslant q-1, i=0, \ldots, n-1$. Then $|F \cap \Delta| \geqslant \sum_{i=1}^{n-1} \epsilon_{i} v_{i-1}$ for any $(n-2)$-space $\Delta$ in $P G(n, q)$ and $|F \cap G|=\sum_{i=1}^{n-1} \epsilon_{i} v_{i-1}$ for some $(n-2)$-spaces $G$ in $P G(n, q)$.

Let $H_{j}, j=1,2, \ldots, q+1$, be the $q+1$ hyperplanes in $P G(n, q)$ that pass through an $(n-2)$-space $G$ intersecting $F$ in $\sum_{i=1}^{n-1} \epsilon_{i} v_{i-1}$ points. Then $F \cap H_{j}$ is a

$$
\left\{\delta_{j}+\sum_{i=1}^{n-1} \epsilon_{i} v_{i}, \sum_{i=1}^{n-1} \epsilon_{i} v_{i-1} ; n-1, q\right\} \text {-minihyper }
$$

in $H_{j}$ for $j=1,2, \ldots, q+1$, where the $\delta_{j}$ are some non-negative integers such that $\sum_{j=1}^{q+1} \delta_{j}=\epsilon_{0}$.

In the case of a $\left\{\delta v_{\mu+1}, \delta v_{\mu} ; n, q\right\}$-minihyper, the parameters in Hamada's theorem become very nice. In the remainder of this article, we will only consider minihypers of this form. The next result of [10] is fundamental for the induction arguments used in the lemmas and theorem which follow.

Lemma 2.5 (Govaerts and Storme [10]) Let $(F, w)$ be a $\left\{\delta v_{\mu+1}, \delta v_{\mu} ; n, q\right\}$-minihyper satisfying $0 \leqslant \delta \leqslant(q+1) / 2,0 \leqslant \mu \leqslant n-1$, and containing a $\mu$-space $\pi_{\mu}$. Then the minihyper $\left(F^{\prime}, w^{\prime}\right)$ defined by the weight function $w^{\prime}$, where

- $w^{\prime}(p)=w(p)-1$, for $p \in \pi_{\mu}$, and

- $w^{\prime}(p)=w(p)$, for $p \in P G(n, q) \backslash \pi_{\mu}$,

is a $\left\{(\delta-1) v_{\mu+1},(\delta-1) v_{\mu} ; n, q\right\}$-minihyper.

It is easy to see that minihypers are closely related to blocking sets. $\mathrm{A}\left\{\delta v_{\mu+1}, \delta v_{\mu} ; n, q\right\}$ minihyper is a $\delta v_{\mu}$-fold blocking set. We state some useful definitions on blocking sets. 
Definition 2.6 A $k$-fold blocking set in $\mathrm{PG}(n, q)$ is a set of points that intersects every hyperplane in at least $k$ points.

A $k$-fold blocking set is called minimal if no proper subset is a $k$-fold blocking set.

A 1-fold blocking set is simply called a blocking set. It is called trivial if it contains a line.

Theorem 2.7 • (Szönyi [16]) A 1-fold blocking set $B$ in $P G(2, q)$, of size $|B|<$ $q+\frac{q+3}{2}$, where $q=p^{h}$, p prime, $h \geqslant 1$, is uniquely reducible to a minimal blocking set $B^{\prime}$ intersecting every line in $1 \quad(\bmod p)$ points.

- (Szönyi and Weiner [17]) A minimal 1-fold blocking set B in PG(n,q), $n \geqslant 3$, $q=p^{h}, p>2$ prime, $h \geq 1$, of size $|B|<q+\frac{q}{2}$, intersects every line in zero points or in $1 \quad(\bmod p)$ points.

\section{Minihypers on the Klein quadric}

It is our intention to prove the non-existence of Cameron-Liebler line classes of parameter $2<x<\frac{q}{2}$ in $\mathrm{PG}(3, q)$ by using $\left\{x\left(q^{2}+q+1\right), x(q+1) ; 5, q\right\}$-minihypers $F$ contained in the Klein quadric $\mathrm{Q}^{+}(5, q)$.

Consider an $\left\{x\left(q^{2}+q+1\right), x(q+1) ; 5, q\right\}$-minihyper $F$, with $x<\frac{q}{2}$, on $\mathrm{Q}^{+}(5, q)$. We know that a hyperplane $H$ intersects $\mathrm{Q}^{+}(5, q)$ in either a parabolic quadric $\mathrm{Q}(4, q)$ or in a tangent cone $\left\langle R, \mathrm{Q}^{+}(3, q)\right\rangle$ with vertex $R$ in $\mathrm{Q}^{+}(5, q)$ and base a 3-dimensional hyperbolic quadric $\mathrm{Q}^{+}(3, q)$.

Lemma 3.1 Let $F$ be an $\left\{x\left(q^{2}+q+1\right), x(q+1) ; 5, q\right\}$-minihyper, with $x<\frac{q}{2}$, contained in the Klein quadric $\mathrm{Q}^{+}(5, q)$, and let $H_{0}$ be a hyperplane in $P G(5, q)$ such that $H_{0} \cap$ $\mathrm{Q}^{+}(5, q)=\left\langle R, \mathrm{Q}^{+}(3, q)\right\rangle$ and such that $H_{0} \cap F$ is an $\{x(q+1), x ; 4, q\}$-minihyper. Then there exists a solid in $H_{0}$, not containing $R$, intersecting $F$ in exactly $x$ points.

Proof First of all, $\left|H_{0} \cap F\right|=x(q+1)<\frac{q^{2}+q}{2}$. Consider a point $R^{\prime}$ of $\mathrm{Q}^{+}(5, q) \cap H_{0}$ with $R^{\prime} \notin F, R^{\prime} \neq R$. There are $q^{3}+q^{2}+q+1$ lines in $H_{0}$ through $R^{\prime}$. At most $\frac{q^{2}+q}{2}$ of them can contain a point of $F$, so there exists a line $l$ through $R^{\prime}$ having an empty intersection with $F$ and not containing $R$. Similarly, we can find a plane $\pi$ through $l$ having an empty intersection with $F$. The $q+1$ solids through $\pi$ together contain $x(q+1)$ points of $F$ and each one of them contains at least $x$ points of $F$ (Theorem 2.4). This means that every solid through $\pi$ contains exactly $x$ points of $F$. Choose one of those solids, not containing $R$, and this is the desired solid.

Lemma 3.2 Let $F^{\prime}$ be an $\{x(q+1), x ; 4, q\}$-minihyper, $x<\frac{q}{2}$, contained in $Q(4, q)$. Then $F^{\prime}$ is the union of $x$ pairwise disjoint lines.

Proof For every point $R \in F^{\prime}$, we find a plane $\pi$ through $R$ only intersecting $F^{\prime}$ in $R$. Then consider all solids through $\pi$, they all contain at least $x-1$ other points of $F^{\prime}$, since every solid contains at least $x$ points of $F^{\prime}$. There remain $x(q+1)-1-(q+1)(x-1)=q$ other points of $F^{\prime}$. So some hyperplane $K_{0}$ through $\pi$ contains more than $x$ points of $F^{\prime}$. 
By [10, Corollary 2], $K_{0} \cap F^{\prime}$ is a blocking set with respect to the planes of $K_{0}$.

Consider the minimal blocking set $B$ inside $K_{0} \cap F^{\prime}$. Suppose that $B$ is not a line.

Take three non-collinear points $R_{1}, R_{2}, R_{3} \in B$. Every line intersects $B$ in zero or in 1 $(\bmod p)$ points $($ Theorem 2.7$)$. The line $l_{1}=\left\langle R_{1}, R_{2}\right\rangle$ already contains two points of $B$, so must contain at least $1+p \geqslant 3$ points of $B$. A line containing more than two points of a quadric lies on that quadric. Similarly, the lines $l_{2}=\left\langle R_{1}, R_{3}\right\rangle$ and $l_{3}=\left\langle R_{2}, R_{3}\right\rangle$ are lines of $\mathrm{Q}(4, q)$. Consider the plane $\pi$ spanned by $l_{1}, l_{2}$ and $l_{3}$. Since these three lines are lines of $\mathrm{Q}(4, q), \pi$ is contained in $\mathrm{Q}(4, q)$, which is impossible.

Thus the minimal blocking set $B$ is a line, hence the minihyper $F^{\prime}$ contains a line $l$. By Lemma 2.5, we have that $F^{\prime} \backslash l$ is an $\{(x-1)(q+1), x-1 ; 4, q\}$-minihyper. Repeating the previous arguments $x$ times gives us that $F^{\prime}$ is the union of $x$ pairwise disjoint lines.

Lemma 3.3 Suppose that $F$ is an $\left\{x\left(q^{2}+q+1\right), x(q+1) ; 5, q\right\}$-minihyper, with $x<\frac{q}{2}$. Suppose that $P$ is a point of $F$ lying on two lines $l_{1}, l_{2}$, completely contained in $F$. Then the plane $\left\langle l_{1}, l_{2}\right\rangle$ is completely contained in $F$.

Proof Suppose that the plane $\left\langle l_{1}, l_{2}\right\rangle \nsubseteq F$, then $F \cap\left\langle l_{1}, l_{2}\right\rangle$ is an $\left\{m_{1}(q+1)+m_{0}, m_{1} ; 2, q\right\}$ minihyper $F^{\prime}$, where $m_{1}+m_{0} \leqslant x<\frac{q}{2}$ (Theorem 2.3). Furthermore, $l_{1} \cup l_{2} \subseteq F$, implying that $\left|\left\langle l_{1}, l_{2}\right\rangle \cap F\right| \geqslant 2 q+1$, which implies $m_{1} \geqslant 2$. So $\left\langle l_{1}, l_{2}\right\rangle \cap F$ is a $t$-fold blocking set, with $m_{1}=t \geqslant 2$. Assume now that $\left|\left\langle l_{1}, l_{2}\right\rangle \cap F\right|=t q+a$, with $a=m_{0}+m_{1} \leqslant x$.

Considering the lines $l_{1}$ and $l_{2}$, and the other $q-1$ lines of $\left\langle l_{1}, l_{2}\right\rangle$ on $P$, we find that $\left|\left\langle l_{1}, l_{2}\right\rangle \cap F\right| \geqslant 2 q+1+(q-1)(t-1)=(t+1) q-t+2$. Hence, $\left|\left\langle l_{1}, l_{2}\right\rangle \cap F\right|=t q+a \geqslant$ $(t+1) q-t+2$, implying $a \geqslant q-t+2$. Now $\left\langle l_{1}, l_{2}\right\rangle \cap F$ is a $t$-fold blocking set of size $t q+a$. Note that $a \leqslant x<\frac{q}{2}$, giving $t \geqslant \frac{q}{2}+2$, a contradiction since $t<\frac{q}{2}$. We conclude that $\left\langle l_{1}, l_{2}\right\rangle \subseteq F$.

\section{Cameron-Liebler line classes and minihypers}

We can now prove the following theorem.

Theorem 4.1 An $\left\{x\left(q^{2}+q+1\right), x(q+1) ; 5, q\right\}$-minihyper, with $x<\frac{q}{2}$, contained in $\mathrm{Q}^{+}(5, q)$ is the union of $x$ pairwise disjoint planes. So for $x \geqslant 3$, such a minihyper does not exist.

Proof ¿From Theorem 2.4, we can find a solid $\Delta$ which intersects $F$ in $x$ points, and such that the $q+1$ hyperplanes through $\Delta$ intersect $F$ in an $\{x(q+1), x ; 4, q\}$-minihyper $F^{\prime}$. These $q+1$ hyperplanes intersect $\mathrm{Q}^{+}(5, q)$ in either a tangent cone or in a non-singular parabolic quadric $Q(4, q)$.

We can make sure that at least $q-1$ hyperplanes through $\Delta$ intersect $\mathrm{Q}^{+}(5, q)$ in non-singular parabolic quadrics. If at least one of them intersects $\mathrm{Q}^{+}(5, q)$ in a tangent cone $\left\langle R, \mathrm{Q}^{+}(3, q)\right\rangle$, Lemma 3.1 says that we can choose $\Delta$ in this hyperplane in such a way that $\Delta$ intersects $\mathrm{Q}^{+}(5, q)$ in a 3-dimensional hyperbolic quadric. The polarity of the Klein quadric then implies that only two hyperplanes through $\Delta$ intersect $\mathrm{Q}^{+}(5, q)$ in tangent cones. 
The $\{x(q+1), x ; 4, q\}$-minihypers $F^{\prime}$ which are the intersection of the other $q-1$ hyperplanes $H_{1}, \ldots, H_{q-1}$ through $\Delta$ with $F$ are contained in non-singular parabolic quadrics and so are the union of $x$ pairwise disjoint lines (Lemma 3.2). Each line of the minihyper $H_{i} \cap F$ intersects $\Delta$ in a point. Suppose that $P$ is a point of $\Delta \cap F$. Then $P$ lies on one line of each minihyper $H_{i} \cap F$, so $P$ lies on at least two lines of the minihyper $F$. From Lemma 3.3 , we know that the plane $\pi$ spanned by these lines is completely contained in $F$. Using Lemma 2.5, we have that $F \backslash \pi$ is an $\left\{(x-1)\left(q^{2}+q+1\right),(x-1)(q+1) ; 5, q\right\}$-minihyper. With $x^{\prime}=x-1<\frac{q}{2}$, we can repeat the previous arguments.

Doing this $x$ times gives us that $F$ is the union of $x$ pairwise disjoint planes. But three planes cannot be pairwise disjoint in $\mathrm{Q}^{+}(5, q)$. So this minihyper does not exist when $x \geq 3$.

We now state the new non-existence results on Cameron-Liebler line classes.

Theorem 4.2 In $P G(3, q), q \geqslant 3$, there exist no Cameron-Liebler line classes with parameter $2<x<\frac{q}{2}$.

Proof Let $\mathcal{L}$ be a Cameron-Liebler line class with parameter $x$. A line $l$ intersects $x(q+1)$ lines of $\mathcal{L}$ if $l \notin \mathcal{L}$ and $l$ intersects $(q+1) x+q^{2}$ lines of $\mathcal{L}$, including $l$, if $l \in \mathcal{L}$ (Definition 1.1).

Translated via the Klein correspondence, $\mathcal{L}$ defines a set $\mathcal{T}$ on $\mathrm{Q}^{+}(5, q)$ such that

$$
\left|P^{\perp} \cap \mathcal{T}\right|= \begin{cases}x(q+1)+q^{2} & \text { if } P \in \mathcal{T} \\ x(q+1) & \text { if } P \notin \mathcal{T}, P \in \mathrm{Q}^{+}(5, q) .\end{cases}
$$

So $\mathcal{T}$ defines an $x$-tight set on $\mathrm{Q}^{+}(5, q)$, with $|\mathcal{L}|=\mathcal{T}=x\left(q^{2}+q+1\right)$. So [1, Theorem 12] implies that $\mathcal{T}$ defines an $\left\{x\left(q^{2}+q+1\right), x(q+1) ; 5, q\right\}$-minihyper $F$ on $\mathrm{Q}^{+}(5, q)$. We only need to check that $\mathcal{T}$ generates $\mathrm{PG}(5, q)$.

Since $|\mathcal{T}| \geq 3\left(q^{2}+q+1\right), \operatorname{dim}\langle\mathcal{T}\rangle \geq 4$. If $\operatorname{dim}\langle\mathcal{T}\rangle=4$, then $\langle\mathcal{T}\rangle \cap \mathrm{Q}^{+}(5, q)=\mathrm{Q}(4, q)$ since $\mathcal{T}$ is not contained in a tangent hyperplane to $\mathrm{Q}^{+}(5, q)$.

Since $|\mathcal{T}|<|\mathrm{Q}(4, q)|$, let $R \in \mathrm{Q}(4, q) \backslash \mathcal{T}$. Consider in $T_{R}(\mathrm{Q}(4, q))$ a plane only intersecting $\mathrm{Q}(4, q)$ in $R$. This plane then lies in the tangent hyperplane $T_{R}(\mathrm{Q}(4, q))$ and in $q$ hyperplanes sharing an elliptic quadric $\mathrm{Q}^{-}(3, q)$ with $\mathrm{Q}(4, q)$.

These elliptic quadrics $\mathrm{Q}^{-}(3, q)$ define via the Klein correspondence regular spreads of $\mathrm{PG}(3, q)$ sharing $x$ lines with $\mathcal{L}$ (Definition 1.1), so these elliptic quadrics contain $x$ points of $\mathcal{T}$. Since $R^{\perp}$ contains $x(q+1)$ points of $\mathcal{T}$, we find that, in total, $\mathcal{T}$ would contain $x(q+1)+x q=2 x q+x$ points. But this is false, since $|\mathcal{T}|=x\left(q^{2}+q+1\right)$.

So, it is indeed true that $\mathcal{T}$ defines an $\left\{x\left(q^{2}+q+1\right), x(q+1) ; 5, q\right\}$-minihyper $F$ on $\mathrm{Q}^{+}(5, q)$. But Theorem 4.1 states that this minihyper does not exist, so we conclude that the Cameron-Liebler line classes with parameter $3 \leq x<\frac{q}{2}$ do not exist.

\section{References}

[1] J. Bamberg, S. Kelly, M. Law, and T. Penttila. Tight sets and $m$-ovoids of finite polar spaces. J. Combin. Theory, Ser. A, to appear. 
[2] J. Bamberg and T. Penttila. Overgroups of cyclic Sylow subgroups of linear groups. Communications in Algebra, submitted.

[3] A. A. Bruen and K. Drudge. On the non-existence of certain Cameron-Liebler line classes in PG(3,q). Des. Codes Cryptogr., 14(2):127-132, 1998.

[4] A. A. Bruen and K. Drudge. The construction of Cameron-Liebler line classes in PG(3,q). Finite Fields Appl., 5(1):35-45, 1999.

[5] P. J. Cameron and R. A. Liebler. Tactical decompositions and orbits of projective groups. Linear Algebra Appl., 46:91-102, 1982.

[6] J. De Beule, K. Metsch, and L. Storme. Characterization results on arbitrary nonweighted minihypers and on linear codes meeting the Griesmer bound. Des. Codes Cryptogr., submitted.

[7] K. Drudge. Extremal sets in projective and polar spaces. PhD Thesis, The University of Western Ontario, 1998.

[8] K. Drudge. On a conjecture of Cameron and Liebler. European J. Combin., 20(4):263-269, 1999.

[9] P. Govaerts and T. Penttila. Cameron-Liebler line classes in PG(3,4). Bull. Belgian Math. Soc., Simon Stevin, 12:793-804, 2005.

[10] P. Govaerts and L. Storme. On a particular class of minihypers and its applications. I: The result for general q. Des. Codes Cryptogr., 28:51-63, 2003.

[11] P. Govaerts and L. Storme. On Cameron-Liebler line classes. Adv. Geom., 4:279-286, 2004.

[12] N. Hamada. A characterization of some $[n, k, d ; q]$-codes meeting the Griesmer bound using a minihyper in a finite projective geometry. Discrete Math., 116(1-3):229-268, 1993.

[13] S. E. Payne. Tight pointsets in finite generalized quadrangles. Congr. Numer., 60: 243-260, 1987.

[14] S. E. Payne. Tight pointsets in finite generalized quadrangles. II. Congr. Numer., $77: 31-41,1990$.

[15] T. Penttila. Cameron-Liebler line classes in PG(3, q). Geom. Dedicata, 37(3):245-252, 1991.

[16] T. Szőnyi. Blocking sets in Desarguesian affine and projective planes. Finite Fields Appl., 3(3):187-202, 1997.

[17] T. Szőnyi and Zs. Weiner. Small blocking sets in higher dimensions. J. Combin. Theory, Ser. A, 95:88-101, 2001. 
Address of the authors: Department of pure mathematics and computer algebra, Ghent University, Krijgslaan 281-S22, 9000 Ghent, Belgium.

J. De Beule: jdebeule@cage.ugent.be, http://cage.ugent.be/ jdebeule

A. Hallez: athallez@cage.ugent.be

L. Storme: ls@cage.ugent.be, http://cage.ugent.be/ ls 